\title{
Time dependent dispersion of nanoparticles in blood vessels
}

\author{
Francesco Gentile ${ }^{1}$, Paolo Decuzzi ${ }^{2}$ \\ ${ }^{1}$ Center of Bio-Nanotechnology and -Engineering for Medicine, Università Magna Graecia, Catanzaro, Italy; \\ ${ }^{2}$ Department of Nanomedicine and Biomedical Engineering, the University of Texas Health Science Center, Houston, USA. \\ Email: gentile@unicz.it
}

Received 25 September 2009; revised 20 October 2009; accepted 25 October 2009.

\begin{abstract}
The dispersion of intravasculary injected nanoparticles can be efficiently described by introducing an effective diffusion coefficient $D_{\text {eff }}$ which quantifies the longitudinal mass transport in blood vessels. Here, the original work of Gill and Sankarasubramanian was modified and extended to include 1) the variation over time of $D_{\text {eff }}$;2) the permeability of the blood vessels and 3) non-Newtonian rheology of blood. A general solution was provided for $D_{\text {eff }}$ depending on space $(\zeta)$, time $(\tau)$, plug radius $\left(\xi_{c}\right)$ and a subset of permeability parameters. It was shown that increasing the vessel plug radius (thus hematocrit) or permeability leads to a reduction in $D_{\text {eff }}$, limiting the transport of nanoparticles across those vessels. It was also shown that the asymptotic time beyond which the solution attains the steady state behaviour is always independent of the plug radius and wall permeability. The analysis presented can more accurately predict the transport of nanoparticles in blood vessels, compared to previously developed models.
\end{abstract}

Keywords: Nanoparticle Transport; Casson Fluid; Permeable Blood Vessels; Drug Delivery

\section{INTRODUCTION}

The study of solute dispersion in capillaries dates back to the celebrated works of Taylor and Aris [1,2], who first studied the effect of shear stress on the transport in laminar flows. They provided a solution for the classic advection/diffusion equation

$$
\frac{\partial C}{\partial t}+\mathbf{u} \cdot \nabla C=D_{m} \nabla^{2} C
$$

in the long term steady state limit, in terms of a constant effective coefficient of diffusion as

$$
D_{e f f}=D_{m}\left(1+\frac{P_{e}^{2}}{192}\right)
$$

which includes the molecular diffusion contribution $(\propto$
$\left.D_{m}\right)$ and the convective contribution $\left(\propto P_{e}\right)$. In the Eqs.1 and 2 above, $P_{e}\left(P_{e}=R_{e} \times \mathrm{u}_{0} / D_{m}\right)$ is the Peclet number for a capillary with radius $R_{e}$ and centerline velocity $\mathrm{u}_{0}$, $C$ is the local solute concentration; $\mathbf{u}$ is the fluid velocity vector; $D_{m}$ is the Brownian or molecular diffusion coefficient and $\nabla$ and $\nabla^{2}$ are the gradient and Laplacian operators, respectively. The solution of Taylor and Aris is valid under the simplifying assumptions of 1) quasi-steady dispersion and 2) unidirectional flow. In particular, it is strictly valid beyond the asymptotic time $t_{s t}=1 / 2 \times R_{e}{ }^{2} / D_{m}$. Notice that sub-micrometric particles with a molecular diffusivity $D_{m}$ typically ranging between $10^{-11}$ and $10^{-9} \mathrm{~m}^{2} / \mathrm{s}$, in large vessels $\left(R_{e} \cong 10^{-2} \mathrm{~m}\right)$ would have $t_{s t}$ of the order of $10^{5}-10^{7} \mathrm{~s}$, whereas in small capillaries $\left(R_{e} \cong 10^{-6} \mathrm{~m}\right) t_{s t}$ would fall in the range $10^{-3}-10^{-1} \mathrm{~s}$.

Considerable efforts were expended in the attempt of relaxing the above assumptions. Gill [3] extended Taylor's formulation to obtain the local concentration $C$ by means of a series expansion about the mean concentration, leading to the Generalized Dispersion Model (GDM), founding upon the rephrased convectivediffusive equation

$$
\frac{\partial \Psi_{m}}{\partial \tau}=\sum_{i=0}^{\infty} K_{i}(t) \frac{\partial^{i} \Psi_{m}}{\partial \varsigma^{i}}
$$

where $K_{i}(t)$ are suitable functions of time; $\Psi_{m}$ is the normalized concentration averaged over a cross section of the capillary as explained in the sequel, $\zeta$ and $\tau$ are the longitudinal and time coordinates respectively. Sankarasubramanian and Gill [4] further developed the GD$M$ including the effect of wall permeability to the solute (i.e. nanoparticles). In 1993, Sharp derived explicit expressions for the constant steady state coefficient $D_{\text {eff }}$ for a non-Newtonian fluid considering, in particular, a Casson-like fluid [5]. Dash et al. [6] and Nagarani et al. [7] combined the model of Sharp and the GDM to obtain the unsteady dispersion in a Casson-like fluid, introducing solute adsorption to the walls. More recently, Decuzzi et al. [8] revisited the theory of Taylor and Aris incorporating the effects of wall permeability for the working fluid (plasma) and deriving a novel and more general 
expression for $D_{\text {eff }}$ being

$$
D_{\text {eff }}=D_{m}\left[1+\frac{P_{e_{0}}^{2}}{192} \times f(\Omega, \Pi, \tilde{z})\right]
$$

where $P_{e_{0}}$ is the Peclet number at the entrance of the capillary $(\widetilde{z}=0)$, and $f$ is a function of the permeability parameter $\Pi$, pressure parameters $\Omega$, and longitudinal coordinate $\widetilde{z}$ along the capillary, as described in the sequel. In 2008, Gentile et al. [9] expanded the solution in [8] to include a Casson-like model for the fluid. Noticeably, the models presented in [8] and [9] are valid in the limit of large times of dispersion or, equivalently, at the steady state. No explicit dependency on time was introduced and the solution was deduced in terms of the longitudinal space coordinate solely.

In this work, the transport formulation proposed in [9] was further developed to account for the time dependency of the problem. The transport of nanoparticles was investigated and the effective diffusion coefficient $D_{\text {eff }}$ derived. $D_{\text {eff }}$ would in general depend upon the permeability of the capillary and the rheology of blood as in [9], but this dependency was extended to all times, thus also comprising the initial regime of dispersion. The model presented herein comprises, in the limits, well established schemes of diffusion.

\section{MATERIALS AND METHODS}

A circular capillary with radius Re and length $l$ was considered as in Figure 1. A Casson-like fluid was considered with capillary walls permeable to the fluid, impermeable and not adsorbent to the solute (i.e. nanoparticles). In the following of the paper, the Generalized Dispersion Model was recalled and revised.

\subsection{The Governing Equations}

Following [4], the dispersion of a solute in a cylindrical capillary was described by the normalized advection-diffusion equation

$$
\frac{\partial \Psi}{\partial \tau}+v \frac{\partial \Psi}{\partial \varsigma}=\left(\frac{1}{\rho} \frac{\partial}{\partial \rho}\left(\rho \frac{\partial}{\partial \rho}\right)+\frac{1}{P_{e_{0}}^{2}} \frac{\partial^{2}}{\partial \varsigma^{2}}\right) \Psi
$$

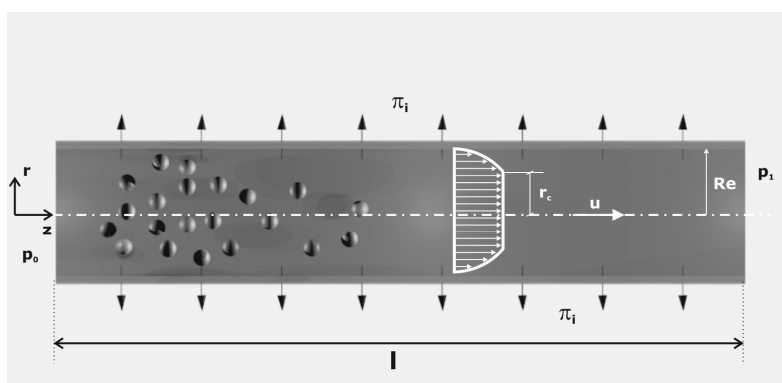

Figure 1. Longitudinal transport of molecules or nanoparticles in a blood capillary with a blunted velocity profile. with the non dimensional terms being

$$
\begin{aligned}
& \Psi=\frac{C}{C_{0}} ; \quad v=\frac{u}{u_{0}} ; \quad \rho=\frac{r}{R_{e}} ; \\
& \varsigma=\frac{D_{m} z}{R_{e}^{2} u_{0}} ; \quad \tau=\frac{D_{m} t}{R_{e}^{2}} ;
\end{aligned}
$$

where $C$ is the local concentration of the solute and $C_{0}$ a reference concentration, $u_{0}$ is the initial center line velocity at the inlet and $u$ the velocity distribution within the capillary with radius $R_{e}, D_{m}$ is the molecular diffusivity of the solute, $r$ and $z$ are the radial and longitudinal coordinates as from the frame of reference in Figure 1, and $t$ stays for the dimensional time. In Eq.6 $P_{e_{0}}\left(=R_{e} \times\right.$ $\left.u_{0} / D_{m}\right)$ is the characteristic Peclet number defined as above. It was assumed that the particles are sufficiently small to have the same velocity of the dislodging fluid so that the diffusion/advection problem and the fluid-dynamic problem may be treated separately. The solution of Eq.5 for $\Psi$ can be derived exactly as

$$
\Psi=\sum_{i=0}^{\infty} f_{i}(\rho, \varsigma ; \tau) \frac{\partial^{i} \Psi_{m}}{\partial \varsigma^{i}}
$$

where the functions $f_{i}$ were related to the $i$-th derivative of $\Psi_{m}$ as shown in the sequel. The mean concentration $\Psi_{m}$ was defined as

$$
\Psi_{m}=2 \int_{0}^{1} \Psi \rho d \rho .
$$

From Eqs.5 and 7, it follows that $\Psi_{m}$ has to satisfy the relation

$$
\frac{\partial \Psi_{m}}{\partial \tau}=\sum_{i=0}^{\infty} K_{i} \frac{\partial^{i} \Psi_{m}}{\partial \zeta^{i}}
$$

where the dispersion coefficients $K_{i}$ were defined properly as function of time as to give

$$
\begin{aligned}
K_{i}(\varsigma, \tau) & =\frac{\delta_{i 2}}{P_{e_{0}}^{2}}+2 \frac{\partial f_{i}}{\partial \rho}(1, \varsigma ; \tau)- \\
& =-2 \int_{0}^{1} f_{i-1}(\rho, \varsigma ; \tau) v(\rho, \varsigma) \rho d \rho
\end{aligned}
$$

with the understanding that $f_{0}=1$ and $f_{1}=0$. Here $\delta_{i 2}$ denotes the delta of Kronecker. The dispersion problem was thus reduced to estimating $f_{i}$ and $K_{i}$ for each $i$. The auxiliary functions $f_{i}$ must satisfy the differential equations

$$
\frac{\partial f_{n}}{\partial \tau}=\frac{1}{\rho} \frac{\partial}{\partial \rho}\left(\rho \frac{\partial f_{n}}{\partial \rho}\right)-v f_{n-1}+\frac{f_{n-2}}{P e_{0}^{2}}-\sum_{i=0}^{\infty} K_{i} f_{n-i} .
$$

Relations Eq.10 and Eq.11 are coupled, and their solution becomes untractable of $i>2$. Nevertheless it was shown [10] that all terms involving a coefficient higher than $i=2$ in Eq.10 can be neglected, in that $K_{2}$ is more than two orders of magnitude greater than $K_{3}$. Eq.9 thus 
reduces to the simplified relation

$$
\frac{\partial \Psi_{m}}{\partial \tau}=K_{1} \frac{\partial \Psi_{m}}{\partial \varsigma}+K_{2} \frac{\partial^{2} \Psi_{m}}{\partial \varsigma^{2}}
$$

where $K_{1}$ and $K_{2}$ represent the convective and diffusive term, respectively. Notice that $K_{i}$ and $f_{i}$ depend upon the velocity field in the capillary $v(\rho, \zeta)$.

\subsection{The Velocity Distribution in Permeable Capillaries}

The velocity distribution in the capillary was given for a Casson-like fluid by [9]

$$
\begin{aligned}
v= & -\frac{d \chi}{d \varsigma} \\
& \times\left\{\begin{array}{lr}
1-\frac{8}{3} \xi_{c}^{1 / 2}+2 \xi_{c}-\frac{1}{3} \xi_{c}^{2} & \text { for } \rho<\xi_{c} \\
-1+\rho^{2}+\frac{8}{3}\left(1-\rho^{3 / 2}\right) \xi_{c}^{1 / 2}-2(1-\rho) \xi_{c} & \text { for } \rho>\xi_{c}
\end{array}\right.
\end{aligned}
$$

where $\xi_{c}$ is the ratio between the plug radius $r_{c}$ and the radius of the capillary $R_{\mathrm{e}}\left(\xi_{c}=r_{c} / R_{\mathrm{e}}\right)$ and $\mathrm{d} \chi / \mathrm{d} \zeta$ is the pressure gradient along $\zeta$. From Eq.13 the non dimensional flow rate was derived through integration over the cross section to give

$$
\Theta=2 \pi \int_{0}^{1} v(r) \rho d \rho=-\frac{\pi}{2} \frac{d \chi}{d \varsigma} A\left(\xi_{c}\right),
$$

where

$$
A\left(\xi_{c}\right)=1-\frac{16}{7} \sqrt{\xi_{c}}+\frac{4}{3} \xi_{c}-\frac{1}{21} \xi_{c}^{4},
$$

and the mean fluid velocity could be written as

$$
\Upsilon=\frac{\Theta}{\pi}=-\frac{1}{2} \frac{d \chi}{d \varsigma} A\left(\xi_{c}\right) .
$$

In the limit of a Newtonian fluid $\left(\xi_{c} \rightarrow 0\right)$, Eq.16 yields the expected value $\Upsilon=0.5$.

In permeable capillaries, the fluid flows laterally across the walls inducing a continuos reduction in mean fluid velocity along the capillary. Following $[8,9]$, the normalized mean fluid velocity $\Upsilon$ was expressed as a function of the hydraulic conductivity $L_{p}$, the interstitial fluid pressure $\pi_{i}$, the inlet and the outlet vascular pressures $p_{0}$ and $p_{1}$, giving

$$
\begin{aligned}
\Upsilon & =-\frac{1}{2} \frac{d \chi}{d \varsigma} A\left(\xi_{c}\right) \\
& =\frac{\cosh \left(\kappa \varsigma \Gamma\left(\xi_{c}\right)\right)-\Omega \cosh \left(\Gamma\left(\xi_{c}\right)-\kappa \varsigma \Gamma\left(\xi_{c}\right)\right)}{1-\Omega \cosh \left(\Gamma\left(\xi_{c}\right)\right)} \frac{A\left(\xi_{c}\right)}{2},
\end{aligned}
$$

where $\Omega$ is a non dimensional pressure parameter

$$
\Omega=\frac{p_{0} / \pi_{i}-1}{p_{1} / \pi_{i}-1}
$$

$\Gamma\left(\xi_{c}\right)$ is the permeability parameter given by

$$
\Gamma\left(\xi_{c}\right)=\frac{4 l}{R_{e}} \sqrt{\frac{\eta}{R_{e}} L_{p}} \frac{1}{\sqrt{A\left(\xi_{c}\right)}}=\frac{\Pi}{\sqrt{A\left(\xi_{c}\right)}} .
$$

Notice that differently from [8], the permeability parameter $\Gamma$ is not fixed and varies with $\xi_{c}$. Substituting back the Eqs.17 and 13 to Eqs.10 and 11, the coefficients $K_{i}$ were appropriately derived.

\subsection{The Initial and Boundary Conditions}

It was assumed that a bolus of nanoparticles is introduced instantaneously and uniformly at the initial time $\mathrm{t}=0$ into the capillary, that is

$$
\Psi(\rho, \varsigma ; 0)=\Psi_{m}(\varsigma ; 0) .
$$

In addition, the walls are impermeable to the solute and no absorption occurs to lead to

$$
\left.\frac{\partial \Psi}{\partial \rho}\right|_{\rho=1}=0
$$

symmetry at the centerline imposed

$$
\left.\frac{\partial \Psi}{\partial \rho}\right|_{\rho=0}=0,
$$

and finally mass conservation was translated in mathematical terms as

$$
\begin{aligned}
\left.\Psi\right|_{\varsigma \rightarrow \infty} & =\left.\frac{\partial^{i} \Psi}{\partial \varsigma^{i}}\right|_{\zeta \rightarrow \infty}=0 ;\left.\quad \Psi_{m}\right|_{\varsigma \rightarrow \infty} \\
& =\left.\frac{\partial^{i} \Psi_{m}}{\partial \varsigma^{i}}\right|_{\varsigma \rightarrow \infty}=0 ; \quad i>0 .
\end{aligned}
$$

The above relations should be also rephrased in terms of $f_{i}$ to solve Eq.11, giving [4,6,7]

$$
\int_{0}^{1} f_{i} \rho d \rho=\delta_{0 i},\left.\frac{\partial f}{\partial \rho}\right|_{\rho=1}=0,\left.\frac{\partial f}{\partial \rho}\right|_{\rho=0}=0 .
$$

\subsection{Solution for $K_{1}$ and $f_{1}$}

Imposing $\mathrm{n}=1$ in Eq.11, it was derived

$$
\frac{\partial f_{1}}{\partial \tau}=\frac{1}{\rho} \frac{\partial}{\partial \rho}\left(\rho \frac{\partial f_{1}}{\partial \rho}\right)-v-K_{1},
$$

and multiplying by $\rho$ and integrating with respect to $\rho$ from 0 to 1, invoking the first of Eq.24, it followed that

$$
K_{1}=-2 \int_{0}^{1} v \rho d \rho=-\Upsilon(\varsigma) .
$$

From Eq.26, it was deduced that the convective term $K_{1}$ equals the mean velocity $\Upsilon$ that is not constant along the capillary. Also notice that assuming a frame of reference moving with $\Upsilon, K_{1}$ would be zero as in [6]. $f_{1}$ was found as a solution of the partial differential Eq.25 
that can be decomposed as the sum the steady state solution $f_{1 s}(\rho, \zeta)$ and the transient term $f_{1 t}(\rho, \zeta ; \tau)$.

$$
f_{1}(\rho, \varsigma ; \tau)=f_{1 s}(\rho, \varsigma)+f_{1 t}(\rho, \varsigma ; \tau) .
$$

Substitution of the steady state term $f_{1 s}$ into Eq.25 yield

$$
\frac{1}{\rho} \frac{\partial}{\partial \rho}\left(\rho \frac{\partial f_{1 s}}{\partial \rho}\right)=v-\Upsilon
$$

which holds in the core of the capillary $\left(\rho<\xi_{c}\right)$, where the velocity is blunted, and in the cell free layer $\left(\rho>\xi_{c}\right)$, where the velocity varies with $\rho$. At the interface, $\rho=\xi_{c}$, continuity imposed that $f_{1 s}\left(\rho=\xi_{c}{ }^{-}\right)=f_{1 s}\left(\rho=\xi_{c}{ }^{+}\right)$which, together with the boundary conditions Eq.24, allowed the deconvolution of $f_{1 s}$ as

$$
\begin{aligned}
f_{1 s}(\rho, \varsigma)= & \frac{\cosh \left(\kappa \varsigma \Gamma\left(\xi_{c}\right)\right)-\Omega \times \cosh \left(\Gamma\left(\xi_{c}\right)-\kappa \varsigma \Gamma\left(\xi_{c}\right)\right)}{1-\Omega \cosh \left(\Gamma\left(\xi_{c}\right)\right)} \\
& \times\left\{\begin{array}{l}
B(\rho) \text { for } \rho<\xi_{c} \\
C(\rho) \text { for } \rho>\xi_{c}
\end{array}\right.
\end{aligned}
$$

where $B$ and $C$ are solely functions of $\rho$ :

$$
\begin{aligned}
& B\left(\rho ; \xi_{c}\right) \\
& =\frac{-8085+21600 \xi_{c}^{1 / 2}-15092 \xi_{c}+1430 \xi_{c}^{4}}{194040} \\
& +\frac{147 \xi_{c}^{6}+1155\left(21-64 \xi_{c}^{1 / 2}+56 \xi_{c}-14 \xi_{c}^{2}+\xi_{c}^{4}\right) \rho^{2}}{194040} \\
& -\frac{2310 \xi_{c}^{4} \ln \left(\xi_{c}\right)}{194040}, \\
& C\left(\rho ; \xi_{c}\right)=-\frac{1}{48}\left(2-6 \rho^{2}+3 \rho^{4}\right) \\
& \quad+\frac{4}{1617}\left(45-154 \rho^{2}+88 \rho^{7 / 2}\right) \xi^{1 / 2} \\
& \quad-\left(7+10 \rho^{2}(2 \rho-3)\right) \xi+\frac{\left(2 \rho^{2}-3\right)}{336} \xi^{4} \\
& \quad+\frac{\xi^{6}}{1320}-\frac{\xi^{4}}{84} \ln (r) .
\end{aligned}
$$

The transient term $f_{1 t}$ depends upon $f_{1 s}$ and was readily derived as [6]

$$
\sum_{n=0}^{\infty} \frac{J_{0}\left(\lambda_{n} \rho\right) f_{1 s}(\rho, \varsigma) \rho d \rho}{J_{0}\left(\lambda_{n}\right)^{2}} e^{-\lambda_{n}^{2} \tau} J_{0}\left(\lambda_{n} \rho\right)
$$

where $J_{0}$ and $J_{1}$ are the Bessel function of first type and order zero and one, respectively, and the eigenvalues $\lambda_{n}$ were found as the roots of the equation $J_{1}\left(\lambda_{n}\right)=0$.

\subsection{Solution for $K_{2}$}

Imposing $\mathrm{n}=2$ in Eq.11, multiplying by $\rho$ and integrat- ing with respect to $\rho$ from 0 to $1, K_{2}$ was obtained as

$$
K_{2}(\varsigma ; \tau)=\frac{1}{P e_{0}^{2}}-2 \int_{0}^{1} f_{1}(\rho, \varsigma ; \tau) v(\rho, \varsigma) \rho d \rho
$$

notice that, differently from the original formulation by Gill and Sankarasubramanian $[4,10]$, the auxiliary functions $K_{2}$ would in general depend also on the longitudinal coordinate $\zeta$ and, in particular, the problem would be determined if the velocity field in the capillary is known. In the limit of large time $K_{2}$ is found as

$$
\begin{aligned}
& \left.K_{2}(\varsigma)\right|_{\tau \rightarrow \infty} \\
= & \frac{1}{P e_{0}^{2}}+\frac{1}{192} \frac{\cosh \left(\kappa \varsigma \Gamma\left(\xi_{c}\right)\right)-\Omega \times \cosh \left(\Gamma\left(\xi_{c}\right)-\kappa \varsigma \Gamma\left(\xi_{c}\right)\right)}{1-\Omega \cosh \left(\Gamma\left(\xi_{c}\right)\right)} \\
& \times\left(1-\frac{5888}{1555} \xi_{c}^{1 / 2}+\frac{558368}{56595} \xi_{c}-\frac{6144}{715} \xi_{c}^{3 / 2}+\frac{128}{45} \xi_{c}^{2}\right. \\
& +\frac{244}{21} \xi_{c}^{4}-\frac{272128}{3773} \xi_{c}^{9 / 2}+\frac{385312}{2205} \xi_{c}^{5}-\frac{4096}{21} \xi_{c}^{11 / 2} \\
& +\frac{11464}{165} \xi_{c}^{6}+\frac{55808}{1155} \xi_{c}^{13 / 2}+-\frac{6976}{165} \xi_{c}^{7}+\frac{430331}{66885} \xi_{c}^{8} \\
& \left.-\frac{512}{147} \xi_{c}^{17 / 2}+\frac{64}{21} \xi_{c}^{9}-\frac{872}{1155} \xi_{c}^{10}+\frac{4}{147} \xi_{c}^{12}-\frac{8}{147} \xi_{c}^{8} \ln \left(\xi_{c}\right)\right)
\end{aligned}
$$

thus recovering the results derived in [9]. Incidentally notice that Eq.34 represents the most general formulation for the non dimensional coefficient of diffusion $K_{2}$ in that it comprises an extensive subset of solutions, depending on the rheological parameters $\xi_{c}, \Gamma$ and $\Omega$. In particular, as $\Gamma$ (or, equivalently, $\Pi$ ) goes to zero (impermeable capillary) Eq.34 coincides with the relation given in [5], whereas as the rheological parameter $\xi_{c}$ goes to zero the result given by [8] is recovered. The classical solution of Taylor and Aris [1,2] is found when both $\Gamma(\Pi)$ and $\xi_{c}$ are null.

\section{RESULTS AND DISCUSSION}

The most important coefficient for estimating the transport of nanoparticles is the normalized effective diffusion coefficient

$$
K_{2}=\left(\frac{D_{e f f}}{D_{m}}-\frac{A\left(\xi_{c}\right)^{2}-1}{A\left(\xi_{c}\right)^{2}}\right) \frac{A\left(\xi_{c}\right)^{2}}{P e_{0}^{2}}
$$

in that it gives a measure of the propensity of the particles to spread about their center of mass along the capillary. Differently from all the schemes proposed so far, the $K_{2}$ presented in Eq.35 changes with $\zeta$ due to the variation of the mean fluid velocity along the permeable vessel. In Figure 2 the relation $192\left(K_{2}-P_{\mathrm{e}}^{-2}\right)$ was plotted as a function of $\zeta$ and $\tau$ in the case of large permeability of the walls $(\Pi=8, \Omega=-2)$ and for a Newtonian 
fluid $\left(\xi_{c}=0\right)$. Generally $K_{2}$ increases with time and attains the steady state value after the early stage of dispersion which corresponds to $\tau=0.5$. A central position of the vessel was observed where $K_{2}-P_{\mathrm{e}}^{-2}=0$, implying that in such area dispersion is solely driven by pure molecular diffusion. The decrease of $K_{2}$ with $\zeta$ strongly depends upon the permeability of the capillary $(\Pi)$ and the plug radius of the fluid $\left(\xi_{c}\right)$. In Figure 3 the 3D plot of the relation $192\left(K_{2}-\right.$ $\left.P_{\mathrm{e}}^{-2}\right)$ as a function of time $\tau$ and position along the capillary $\zeta$ was displayed showing the effects of $\Pi$ and $\zeta_{c}$ varying between 0 and 4 and 0 and 0.4 respectively, and for a constant $\Omega=-2$. In Figure 4, the contourplots corresponding to Figure 3 were reported. As time increases, the solution for $K_{2}$ tends to a constant asymptotic value. Noticeably, the time beyond which dispersion turns to be time independent is always less than 0.5 , regardless $\Pi$ and $\xi_{c}$ Therefore, the permeability parameter and the plug radius have a negligible effect upon the process of diffusion along with time but do effect on the steady state behavior of the system. In particular, when both $\Pi$ and $\xi_{c}$ are larger than zero the reduction in dispersion $\left(D_{\text {eff }}\right.$ or $\left.K_{2}\right)$ is dramatic, and in large portions of the capillary the transport of the nanoparticles is mostly diffusion limited. This is easily explained observing that longitudinal transport is enhanced by radial velocity gradients (shear diffusion), thereby either an increase of the core region of the capillary with a flat velocity profile (thus $\xi_{c}$ ) or a reduction in the velocity amplitude due to an augmented permeability (thus $\Pi$ ), generates a decrease in $K_{2}$, as thoroughly discussed in $[8,9]$.

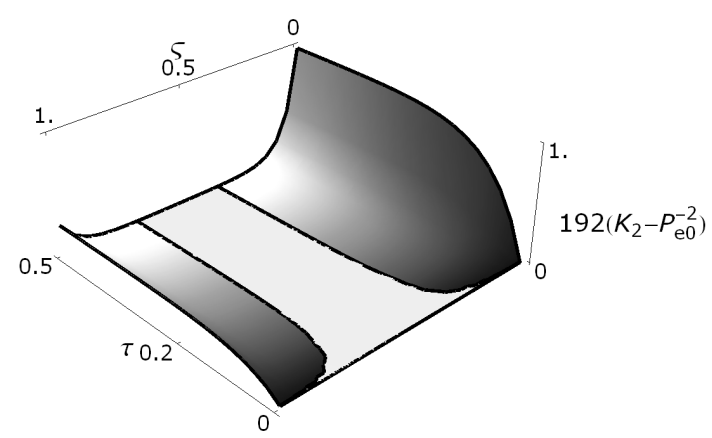

Figure 2. The dimensionless effective diffusion $K_{2}$ as a function of the normalized position $(\zeta)$ and time $(\tau)$ for a fixed plug radius $\xi_{c}=0$ and for a permeable capillary $(\Pi=8, \Omega=-2)$.
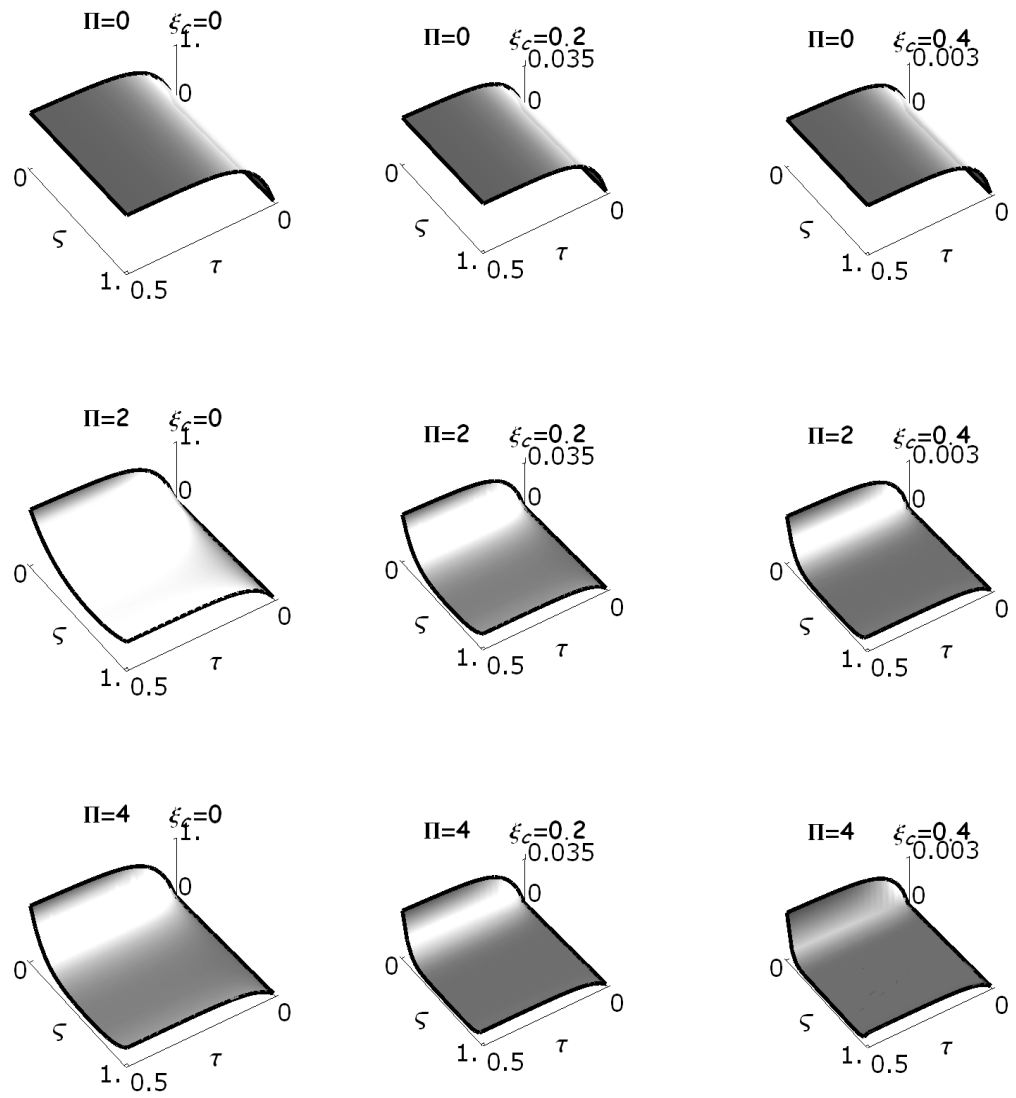

Figure 3. 3D plots of the dimensionless effective diffusion coefficients $K_{2}$ as a function of the normalized position $(\zeta)$ and time $(\tau)$, for $\Pi$ and $\xi_{c}$ varying between 0 and 4 , and 0 and 0.4 respectively, and for a constant $\Omega=-2$. 

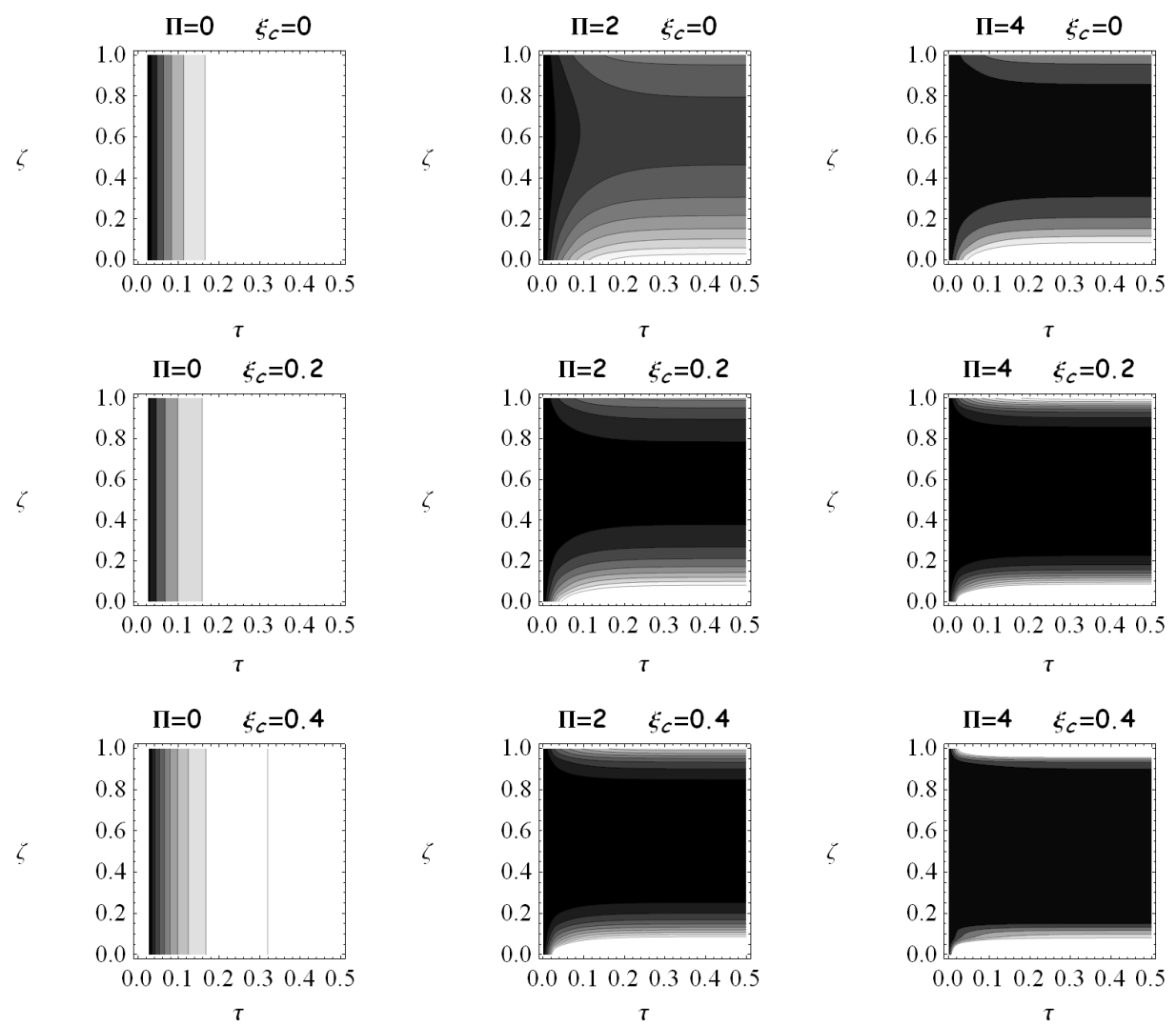

Figure 4. Contour plots of the dimensionless effective diffusion coefficients $K_{2}$ as a function of the normalized position $(\zeta)$ and time $(\tau)$, for $\Pi$ and $\xi_{c}$ varying between 0 and 4 , and 0 and 0.4 respectively, and for a constant $\Omega=-2$.

Given $K_{2}$, the effective diffusion coefficient $D_{\text {eff }}$ was deduced as

$$
D_{e f f}=\frac{R_{e}^{2} u_{0}^{2}}{A\left(\xi_{c}\right)^{2} D_{m}} K_{2}+\frac{A\left(\xi_{c}\right)^{2}-1}{A\left(\xi_{c}\right)^{2}} D_{m},
$$

or, equivalently

$$
\frac{D_{e f f}}{D_{m}}=\frac{P_{e_{0}^{2}}^{2}}{A\left(\xi_{c}\right)^{2}} K_{2}+\frac{A\left(\xi_{c}\right)^{2}-1}{A\left(\xi_{c}\right)^{2}} .
$$

Eq.37 shows that any enhancement in effective diffusion over the Brownian diffusion $\left(D_{m}\right)$ is proportional to the product $P_{e_{0}} \times K_{2}$ and would strongly depend on the local hydrodynamics and capillary size.

The dimensionless effective diffusion $D_{e f f} / D_{m}$ as a function of the rheological parameter $\xi_{c}$, for different values of $P_{e}$ and for a fixed $\Pi=0$ was shown in Figure 5. As expected, confirming the results derived in [9], larger $P_{e}$ and smaller $\xi_{c}$ lead to larger $D_{e f f} / D_{m}$ ratios. Figure 6 illustrated the ratio $D_{e f f} / D_{m}$ over time, for an impermeable channel $(\Pi=0)$ and for different values of $\xi_{c}$. Figure 7 reported the same diagram of Figure 6 for a permeable channel $(\Pi=2$, $\Omega=-2$ ). In all cases, a steady state value was attained for $\tau$ larger than 0.5 . Notice that for $\Pi=0$ and for $\xi_{c}$ moving from 0 to 0.4 , at large times the classical solutions of Taylor and Aris [1,2] $\left(\xi_{c}=0\right)$, and Sharp [4] $\left(\xi_{c}=0.2,0.4\right)$ are recovered (Figure 6). When the permeable solution was instead considered (Figure 7), the steady state values recapitulated the results given by Decuzzi et al. [8] $\left(\xi_{c}=0\right)$ and Gentile et al. [9] ( $\left.\xi_{c}=0.2,0.4\right)$.

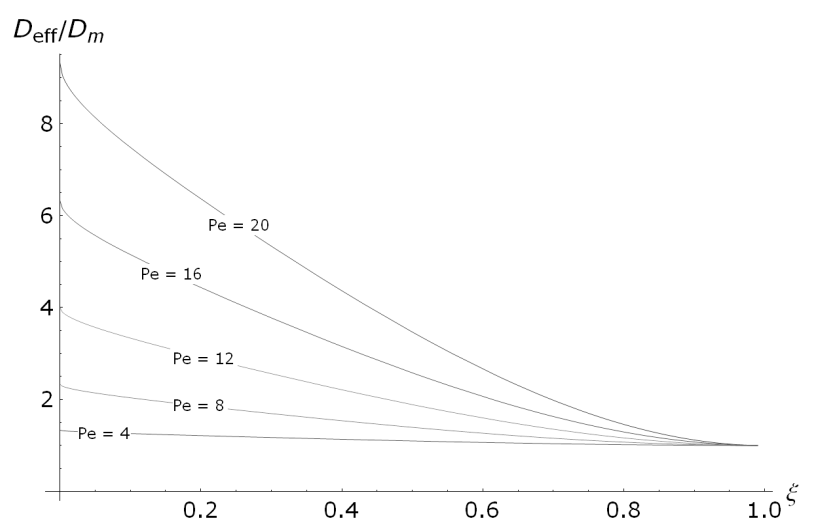

Figure 5. The dimensionless effective diffusion $\left(D_{\text {eff }} / D_{m}\right)$ as a function of the rheological parameter $\xi_{c}$, for different values of Pe and for a fixed $\Pi=0$. 
$\Pi=0$

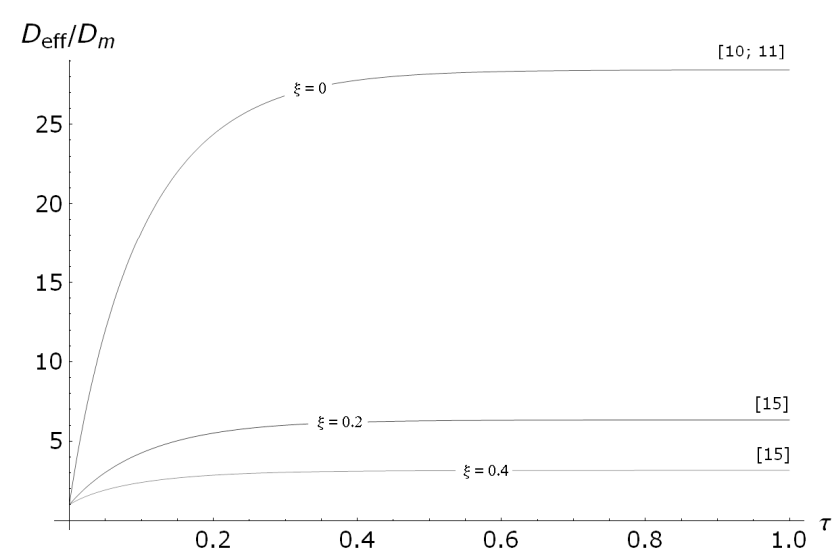

Figure 6. The ratio $\left(D_{\text {eff }} / D_{m}\right)$ over time, for a permeable channel $(\Pi=0)$ and for different values of $\xi_{c}$.

$\Pi=2 \Omega=-2$

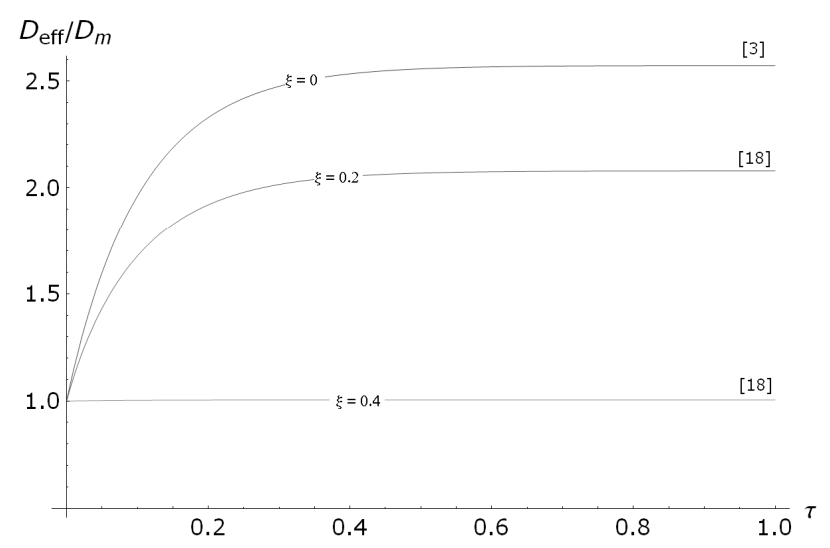

Figure 7. The ratio $\left(D_{\text {eff }} / D_{m}\right)$ over time, for a permeable channel $(\Pi=2 ; \Omega=-2)$ and for different values of $\xi_{c}$.

Table 1. Average dimensions and velocities of blood vessels (Decuzzi, 2006 [8]). $P_{e}$ is calculated for $D_{m}=6 \times 10^{-13} \mathrm{~m}^{2} / \mathrm{s}$.

\begin{tabular}{ccccc}
\hline Vessel & $\mathrm{L}[\mathrm{mm}]$ & $\mathrm{R}_{\mathrm{e}}[\mathrm{mm}]$ & $\mathrm{U}[\mathrm{mm} / \mathrm{s}]$ & $\boldsymbol{P}_{e}$ \\
\hline Aorta & 50 & 25 & 400 & $1.6 \times 10^{10}$ \\
Artery & $1.5-2$ & 4 & 100 & $6.67 \times 10^{8}$ \\
Arteriole & $1.5-2$ & $0.02-0.1$ & 5 & $1.67-8.33 \times 10^{5}$ \\
Capillary & 0.5 & $0.005-0.001$ & $0.1-1$ & $833-41667$ \\
Venules & 1 & $0.02-0.05$ & 0.5 & $1.66-4.16 \times 10^{4}$ \\
Vein & $1-14$ & $2-5$ & 50 & $1.6-4.1 \times 10^{8}$ \\
Vena & $40-50$ & 30 & 100 & $5 \times 10^{9}$ \\
Cava & & & & \\
\hline
\end{tabular}

Recalling that the width of the plug radius $\xi_{c}$ scales with $R_{e}$ as $\xi_{c} \sim 1-3 \times R_{e}^{-0.8}[9]$ and considering the data of
Table 1, moving from capillaries to arterioles and venules $\mathrm{P}_{0}$ significantly increases and the ratio $D_{\text {eff }} / D_{m}$ augments accordingly despite a reduction of the cell free layer area. Figure 8 showed the minimum value that $D_{e f f} / D_{m}$ would assume in a vessel at the steady state as a function of Re and of the plug radius $\xi_{c}$ for an impermeable vessel $(\Pi=0)$. Figure 9 reported the same diagram for a permeable vessel $(\Pi=5, \Omega=-2)$. It was observed

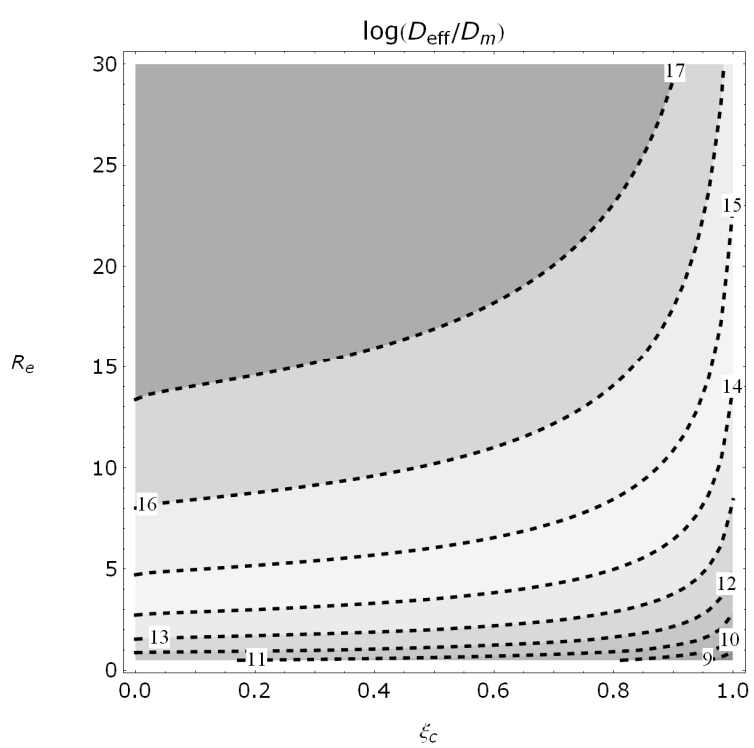

Figure 8. The minimum value that $D_{\text {eff }} / D_{m}$ would assume in a vessel at the steady state as a function of Re and of the plug radius $\xi_{c}$; for an impermeable vessel $(\Pi=0)$.

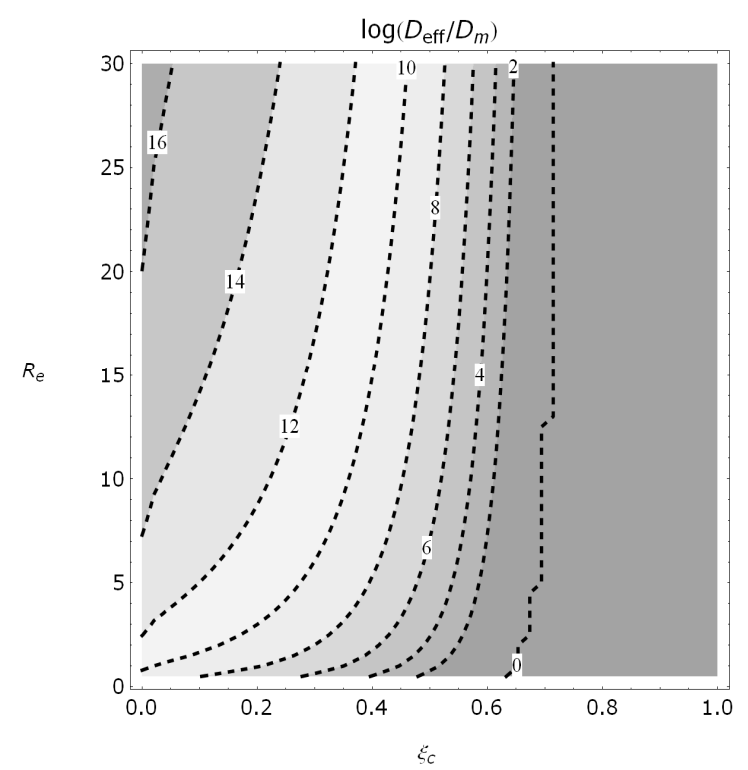

Figure 9. The minimum value that $D_{\text {eff }} / D_{m}$ would assume in a vessel at the steady state as a function of Re and of the plug radius $\xi_{c}$; for a permeable vessel $(\Pi=5$, $\Omega=-2$ ). 
that the effect of the radius of the vessel (or equivalently of $P_{\mathrm{e} 0}$, see Table 1) dominates over that of the plug radius, meaning that in large capillaries, where $\xi_{c}$ is large, yet the longitudinal diffusion increases up to $10^{6}$ times with respect to small vessels. And this effect is dramatically amplified considering leaky or fenestrated capillaries. It was argumented in $[8,9]$ that in a capillary network passively transported molecules or nanoparticles would follow the path with the largest effective diffusion. Therefore, nanoparticles and molecules would in a larger percentage stay in the macrocirculation (high $D_{\text {eff }}$ ) rather than in the microcirculation (small $D_{e f f}$ ) or highly permeable vessels (even smaller $D_{\text {eff }}$ ), as for instance in the angiogenic tumor vasculature. This would constitute a barrier to the rational systemic administration of therapeutic and contrast agents. The correct design of nanoparticles could constitute an effective way to overcome this barrier. It was demonstrated, either experimentally [11-13] and theoretically [14], that particles having different sizes or shapes also have different margination properties, that is the attitude of "spontaneously" drifting towards the walls of the blood vessels. In particular, in considering the case of spherical particles, it was shown that the delivery efficiency is not size independent, instead larger particles would perform better than smaller ones under the effect of a gravitational or magnetic field [11]; whereas, below a characteristic diameter, the settling of nanoparticles would be mostly size independent [13]. In considering shapes other than spherical, it was demonstrated that, in the range of physiological relevant values of shear rates, inertial discoidal particles perform better than quasi-hemispherical and significantly better than spherical, and this circumstance would suggest the use of discoidal carriers in drug delivery $[12,14]$.

In sight of the above findings, it is understandable that tailoring the shape and size of nanovectors inasmuch that they would tend to accumulate in the 'cell free layer', could significantly increase the efficiency of delivery.

\section{CONCLUSIONS}

The Generalized Dispersion Model firstly introduced by Gill and Sankarasubramanian was revised to account for blood rheology and vessel permeability. The non dimensional coefficient of diffusion was derived as a function of time, of the plug radius $\xi_{c}$ and of a subset of permeability parameters, $\Pi$ and $\Omega$. It was observed that an enhancement in permeability or a blunted velocity profile (high hematocrit) dramatically reduces vascular transport. It was seen that an augmented permeability at the vessels walls does not influence the time in correspondence of which the dispersion process attains the steady state. Evidence was given that freely administrated drugs or nanoparticles very harshly would leave the macrocirculation in favour of leaky capillaries of tumor districts. Strategies for the avoidance of this physiological barrier were proposed.

\section{REFERENCES}

[1] Taylor, G. (1953) Dispersion of soluble matter in solvent flowing slowly through a tube. Proceedings of the Royal Society of London, A, 219(1137), 186-203.

[2] Aris, R. (1956) On the dispersion of a solute in a fluid flowing through a tube. Proceedings of the Royal Society of London, A, 235(1200), 67-77.

[3] Gill, W.N. (1967) A note on the solution of transient dispersion problems. Proceedings of the Royal Society of London, A, 298(1967), 335-339.

[4] Sankarasubramanian, R. and Gill, W.N. (1973) Taylor diffusion in laminar flow in an eccentric annulus. Proceedings of the Royal Society of London, A, 333, 115-132.

[5] Sharp, M.K. (1993) Shear-augmented dispersion in nonNewtonian fluids. Annals of Biomedical Engineering, 21(4), 407-415.

[6] Dash, R.K., Jayaraman, G. and Mehta, K.N. (2000) Shear augmented dispersion of a solute in a casson fluid flowing in a conduit. Annals of Biomedical Engineering, 28(4), 373-385.

[7] Nagarani, P., Sarojamma, G. and Jayaraman, G. (2004) Effect of boundary absorption in dispersion in casson fluid flow in a tube. Annals of Biomedical Engineering, 32(5), 706-719.

[8] Decuzzi, P., Causa, F., Ferrari, M. and Netti, P.A. (2006) The effective dispersion of nanovectors within the tumor microvasculature. Annals of Biomedical Engineering, 34(4), 633-641.

[9] Gentile, F., Ferrari, M. and Decuzzi, P. (2008) The transport of nanoparticles in blood vessels: The effect of vessel permeability and blood rheology. Annals of Biomedical Engineering, 2(36), 254-261.

[10] Gill, W.N. and Sankarasubramanian, R. (1970) Exact analysis of unsteady convective diffusion. Proceedings of the Royal Society of London, A, 316, 341-350.

[11] Decuzzi, P., Gentile, F., Granaldi, A., Curcio, A. and Indolfi, C. (2007) Flow chamber analysis of size effects in the adhesion of spherical particles. International Journal of NanoMedicine, 2(4), 1-8.

[12] Gentile, F., Chiappini, C., Fine, D., Bhavane, R.C., Peluccio, M.S., Cheng, M., Liu, X., Ferrari, M. and Decuzzi, P. (2008) The margination dynamics of non spherical inertial particles in a microchannel. Journal of BioMechanics, 41(10), 2312-2318.

[13] Gentile, F., Curcio, A., Indolfi, C., Decuzzi, P. and Ferrari, M. (2008) The margination propensity of spherical particles for vascular targeting in the microcirculation. Journal of Nanobiotechnology, 6(9), 1-9.

[14] Lee, S.Y., Ferrari, M. and Decuzzi, P. (2009) Design of bio-mimetic particles with enhanced vascular interaction. Journal of Biomechanics, 42(12), 1885-1890. 